\title{
loT framework and requirement for intelligent industrial pyrolysis process to recycle CFRP composite wastes: application study
}

This paper was downloaded from TechRxiv (https://www.techrxiv.org).

\section{LICENSE}

CC BY 4.0

SUBMISSION DATE / POSTED DATE

$12-11-2021 / 29-11-2021$

\section{CITATION}

Ullah, Mehar; Gopalraj, Sankar Karuppannan; Rojas, Daniel Gutierrez; Nardelli, Pedro; Karki, Timo (2021): IoT framework and requirement for intelligent industrial pyrolysis process to recycle CFRP composite wastes: application study. TechRxiv. Preprint. https://doi.org/10.36227/techrxiv.16995043.v2

$\mathrm{DOI}$ 


\title{
IoT framework and requirement for intelligent industrial pyrolysis process to recycle CFRP composite wastes: application study
}

\author{
Mehar Ullah ${ }^{10000-0003-2405-8998]}$, Sankar Karuppannan Gopalraj ${ }^{2}$, Daniel Gutierrez-Rojas ${ }^{1}$, Pedro Nardelli ${ }^{1}$ and Timo Kärki ${ }^{2}$ \\ ${ }^{1}$ School of Energy Systems, LUT University, Yliopistonkatu 34, 53850 Lappeenranta, Finland \\ ${ }^{2}$ Fiber Composite Laboratory, Department of Mechanical Engineering, LUT University, P.O. Box 20, 53850 Lappeenranta, Finland. \\ Mehar.Ullahelut.fi
}

\begin{abstract}
The cumulating carbon fiber-reinforced polymer (CFRP) composite wastes need to be disposed efficiently. So far, the most effective thermal-based recycling technique, namely pyrolysis, has grown exponentially towards industrial scaling in developed countries such as the UK and Germany. Typically, even the slightest mistakes can cause unfavorable results and delays in workflow within such a massive operating environment (e.g., $>1$ tonnes/day operating capacity). The existing semi-automated and, in some cases, fully automated plants should be continuously updated to resemble the varying classes and volume of the CFRP composite wastes. To overcome such research gaps and imprecise manual errors, Internet-of-Things (IoT) based framework is proposed. This paper studies the theoretical implementation of an IoT-based framework into the pyrolysis process to recycle CFRP composite waste to manage the process based on the principles of cyber-physical systems. The proposed framework consists of sensors and actuators that will be used to collect the data and communicate with a central management constructed as a platform that will articulate and manipulate data to satisfy the requirements of the recycling process, computationally modeled through logical relations between physical entities. In this case, the management unit can be either controllable or monitored remotely to increase the operation time of the plant. Our objective is to propose a scalable method to improve the recycling process, which will also help future decision-making in handling recycled carbon fiber. Specifically, this study will go beyond the state-ofthe-art in the field by (i) automatically calculate the mass of the waste and adjust the operating time, temperature, atmospheric pressure, and inert gas flow (if needed), (ii) regenerating heat so that after the first batch is recycled, the resin high in calorific value will be burned and will be releasing energy, whose generated heat needs to be trapped inside the furnace and then regenerated into the system, and (iii) decrease energy consumption and fasten the process flow time. In summary, the proposed framework aims to provide a user-friendly control and temperature monitoring that can increase the overall efficiency of the process and avoid possible process shut down or even char formation by a controlled atmosphere in the pyrolytic reactor.
\end{abstract}

Keywords: IoT Framework, Industrial-scale Pyrolysis, Recycling Carbon Fiber.

\section{$1 \quad$ Introduction}

Carbon fiber-reinforced polymer (CFRP) composites have been exponentially used in high-performance applications for decades. The composites have high mechanical properties for a lower weight ratio making them capable of replacing traditional metals in lightweight applications. However, CFRP composites employed 20 years ago have now reached their end-of-life (EoL) and raised a significant question about their disposal routes. So far, 62000 tonnes of CFRP composite wastes have been cumulating each year, and the forecast predicts that the amount could increase up to $90,000+$ tonnes/year if not disposed properly. At the same time, the annual demand for virgin CFRP composites also expected to be increased from 72,000 to 140,000 tonnes/ year [1]. To establish a balance, recycling the waste composites, recovering the valuable carbon fibers (CFs), and reusing them into new composites is the only sustainable option [2].

Previously, landfilling and incineration were the popular disposal methods for their composites. However, various studies have proved that recycled carbon fibers ( $\mathrm{rCFs}$ ) to be close to their virgin properties, recycling industries have invested in a sustainable alternative to recycling CFRP composite wastes. Thermal recycling processes such as pyrolysis 
and solvolysis using supercritical/ subcritical water or mild solvents have proved to be highly efficient [1]. Recently, a novel thermal recycling process [2] capable of recycling CFRP composite wastes with maximum efficiency has resulted in clean recycled fibers without disturbing the fibers' structural integrity (fiber direction, arrangement, and length). However, all these processes exist on a laboratory scale. Among them, pyrolysis has been successfully established on an industrial scale in The UK (Gen 2 Carbon), Belgium (Procotex), and Germany (CFK valley \& SGL group).

Fig. 1 presents the operating principle of the pyrolysis recycling process to recover CFRP composite wastes. First, the composite waste is size reduced using mechanical shredding and feeds into the system. The pyrolysis reactor is a closed chamber with no oxygen present. However, the process is done in an inert gas atmosphere to separate the valuable CFs from the matrix at $550{ }^{\circ} \mathrm{C}$ for the required time (depends on the waste quantity). Then, the recovered fibers are passed to a secondary heating chamber at $200{ }^{\circ} \mathrm{C}$ to oxidize the resin residues. Finally, the CFs are recycled, acquiring pyrolytic oil (can be used as feedstock) and hot gas (can be regenerated) [1]. Overall, industrial-scale pyrolysis possesses enormous sustainable benefits.

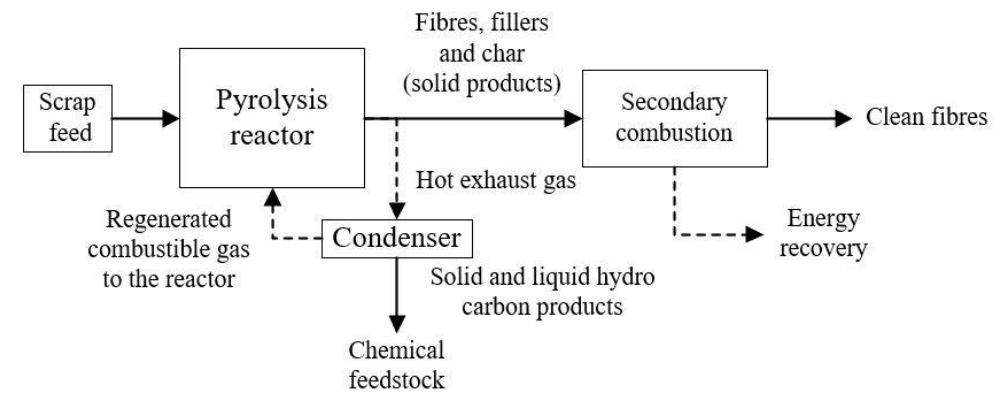

Fig. 1. Overview of the pyrolysis process [1]

The EU's regulations have moved from landfilling and incineration towards sustainable recycling regulations. In which recycling processes with low emissions in carbon footprints are preferred [1]. So, industries utilizing the pyrolysis process to recycle CFRP composite waste have to reconsider advanced emission monitoring along with their primary monitorable parameters such as pyrolysis temperature, pressure, inert gas flow, heat regeneration, recycled fiber quality, etc. Also, considering the type of CFRP composite wastes cumulating, which lacks composite information such as profile, the volume of fiber and resin, composite type, resin type, etc., there is a need to implement an advanced monitoring system to moderate such industrial-scale process.

These strategies call for more control and automation. Therefore, applying IoT scheme frameworks can improve the overall connectivity bringing significant control, monitoring, and safety to the process [3]. However, the existing semiautomated and, in some cases, fully automated pyrolysis plants need to be continuously updated and constantly monitored to couple with the varying CFRP composite waste types. To overcome such research gaps and eradicate manual errors, Internet-of-Things (IoT) based framework is proposed. Typically, an IoT is a network of devices interconnected to each other using some communication technology and using sensors and actuators to gather data from different devices and send that data to the cloud to store, process, and get information [4].

This paper studies the theoretical implementation of an Internet-of-Things (IoT)-based framework into the pyrolysis process to recycle CFRP composite wastes and manage the process based on the principles of cyber-physical systems. The proposed framework consists of sensors and actuators to collect the required data and communicate with central management constructed as an IoT platform that will articulate and manipulate data to satisfy the requirements of the recycling process, computationally modeled through logical relations between physical entities. Furthermore, this study will focus on selecting a suitable IoT platform based on the requirements of the pyrolysis process. Incorporating IoT and its platform into the pyrolysis process will improvize the plant by automatically calculating the mass of the CFRP composite 
wastes and adjusting parameters such as: operating time, temperature, atmospheric pressure, inert gas flow, increase energy efficiency, and reduce process flow time.

\section{General IoT framework for recycling carbon fibers}

The focus on implementing IoT technology into industrial-scale pyrolysis was discussed in the following section. First, an insight into improving pyrolysis factors such as cost, speed, reliability, scalability, and performance resulting from IoT was investigated [5]. Furthermore, discussions on IoT collected data as a reliable source of information in current decisionmaking and future improvements into the pyrolysis process.

\subsection{Industrial-scale pyrolysis process and IoT framework}

Fig. 2 presents the implemented IoT network in an industrial-scale pyrolysis setup. The process includes sensors and actuators at all the crucial sections (starting from the waste feed till the CF recovering) throughout the process. The IoT framework is designed to be flexible and extended to multiple furnaces (pyrolysis process 1 , pyrolysis process $2 . . . .$. pyrolysis process N). Fig. 1 presents the pyrolysis process setup. The gathered data from the individual furnaces are sent to the main gateway and forwarded to the cloud for storage, processing, and visualization. In addition, information can be taken from data stored in the cloud. Based on that data, a further future business decision can be taken that can improve and speed up the CF recycling process with precision and accuracy.

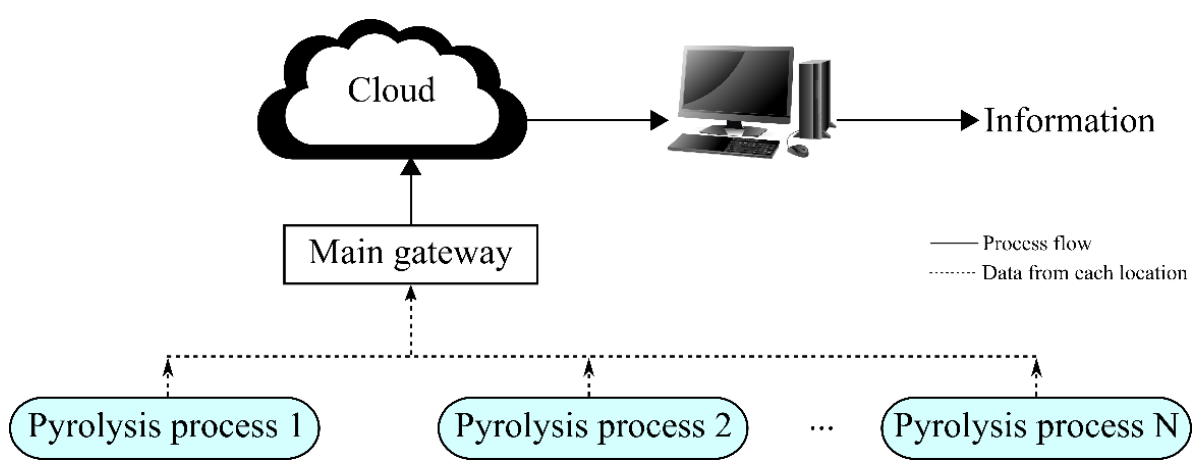

Fig. 2. IoT framework implementation in industrial-scale pyrolysis

\subsection{The role of IoT in process monitoring}

CFRP waste recycling process will be benefited from an IoT deployment by adding physical elements to the cyberworld on what is known as a Cyber-Physical System (CPS), as introduced in [4]. This will enable better control of the input material transported along with the conveyor, control temperature of the furnace, and real-time monitoring of char production. In Fig. 3, It can be seen how IoT is used to form a cyber-physical environment where sensing and actuators are connected to the gateway making the interface to the cloud server. In the cyber world, a manager can remotely perform monitoring, control, or analyze stored data. 


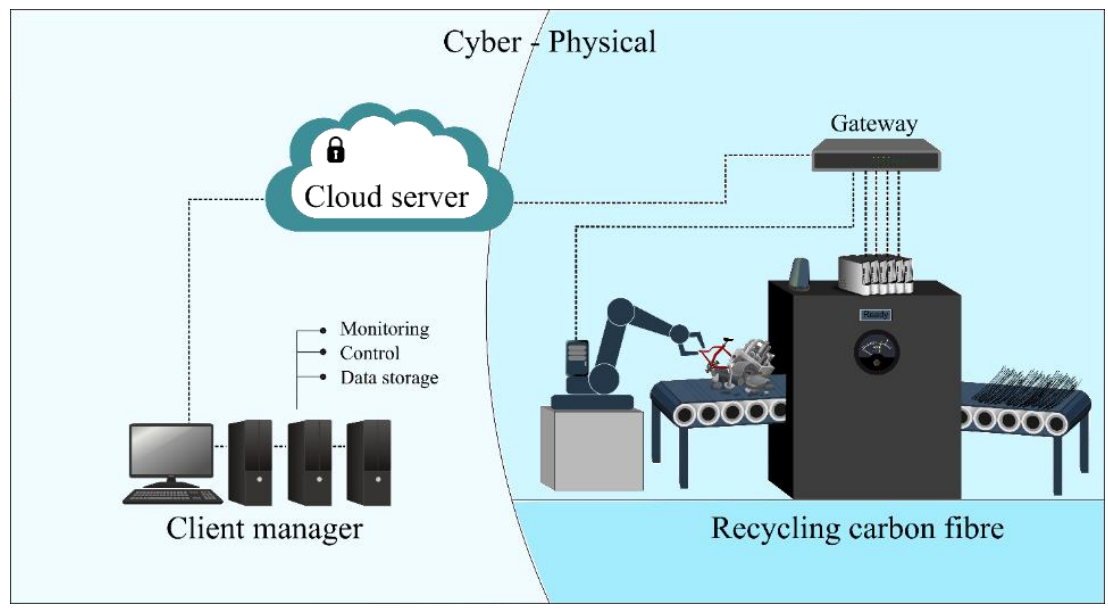

Fig. 3. IoT schematic of an in-site CF recycling process

\subsection{Selection of IoT platform for pyrolysis process}

Fig. 4 presents the framework of IoT platforms. IoT application needs a platform to run smoothly and provide the data to make future decisions based on the data received from the IoT platform. Hundreds of IoT platforms are available, and finding a suitable IoT platform for a specific IoT application is complex. A lack of experience and knowledge compounds the problem, and in some cases, a company may select a platform without adequate requirements analysis, which later leads to problems [6]. Companies can select an appropriate IoT platform for their IoT application if they first analyze their business requirements and start selecting the IoT platform with precise business requirements and knowing key factors of IoT platforms [5].

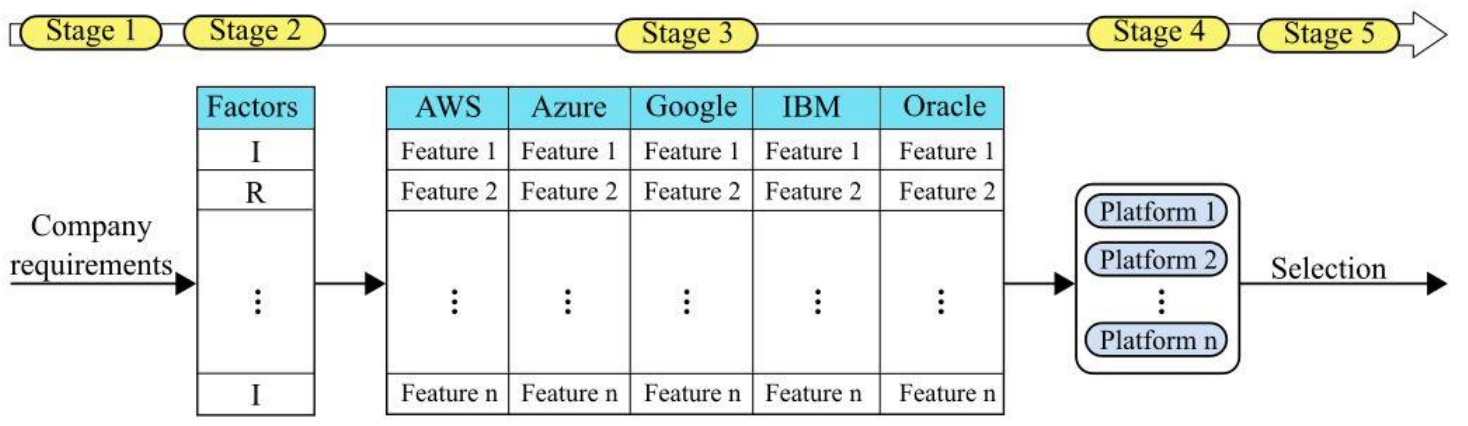

Fig. 4. Framework for the selection of the IoT platform

The process for the selection of an IoT platform has five stages. In stage 1, the company identify and finalizes their business requirements. In stage 2, the identified requirements are categorized as "Important" or "Required". In stage 3, the requirements are compared with the features provided by the IoT platforms. Only the five most important IoT platforms based on the market shares are selected in this case. In stage 4, IoT platforms are selected that are capable of fulfilling the requirements. Finally, in stage 5, a suitable platform is selected for the business IoT application.

The requirements for the pyrolysis process to recycle CFRP composite waste are to eliminate the manual work involved in the process, speed up the recycling time (> 1 tonnes/day), increase energy efficiency, and increase the overall recycling efficiency of the process. Furthermore, it calculates the amount of heat required for a specific amount of CFRP composite 
waste and estimates the amount of heat generated during the process. Finally, calculates the amount of emissions (exhaust and later outside the system). In short, the pyrolysis process needs stability, flexibility, scalability, security, attractive interface, data analytics, and interoperability throughout the system process. The process also needs and user-friendly application development environment for its IoT business application in the cloud.

The requirements of the pyrolysis process are categorized into "Important" and "Required" factors. The requirements stability, flexibility, scalability, security, attractive interface, and data analytics are considered "Important", and the requirements interoperability is considered "Required". The requirements of the pyrolysis process are compared with the features provided by the selected five major IoT platforms, as shown in Table 1. All the essential requirements of the pyrolysis process were matching with the features provided by the AWS IoT platform. However, Azure lacked the required flexibility and the required factor Interoperability.

Table 1. IoT platform features adopted from [5]

\begin{tabular}{|c|c|c|c|c|c|}
\hline Factors & AWS & Azure & $\begin{array}{l}\text { Google } \\
\text { Cloud }\end{array}$ & $\begin{array}{l}\text { IBM } \\
\text { Watson }\end{array}$ & $\begin{array}{l}\text { Oracle } \\
\text { IoT }\end{array}$ \\
\hline Scalability & Yes & Yes & yes & yes & yes \\
\hline Flexibility & Yes & - & yes & - & yes \\
\hline Data analytics & Yes & yes & yes & yes & yes \\
\hline Disaster recovery & Yes & yes & no & no & no \\
\hline Stability & Yes & yes & yes & - & - \\
\hline Security & high & high & high & high & high \\
\hline Data ownership & - & yes & - & - & - \\
\hline Protocol support & Yes & yes & - & yes & yes \\
\hline System performance & Yes & - & yes & yes & - \\
\hline Time to market & Yes & yes & - & - & yes \\
\hline Legacy architecture & Yes & - & - & - & yes \\
\hline Attractive interface & Yes & yes & - & no & - \\
\hline Pricing model & bad & bad & good & - & - \\
\hline Cloud ownership & Yes & yes & yes & - & yes \\
\hline Interoperability & Yes & - & - & - & yes \\
\hline App. environment & Yes & yes & yes & yes & yes \\
\hline Hybrid cloud & Yes & yes & - & - & - \\
\hline Platform migration & Yes & yes & - & - & - \\
\hline Previous experience & Yes & yes & - & - & - \\
\hline Edge intelligence & Yes & yes & yes & - & yes \\
\hline Bandwidth & - & - & good & - & - \\
\hline
\end{tabular}

\subsection{Communication requirements}

Currently, wireless communication advances have countless open opportunities for industrial applications. They can be critical enablers for monitoring and controlling impossible tasks before due to low flexibility and cost [7]. For the past decades, requirements for the industrial-scale process have been discussed, but they are bounded to every application. 5G cellular communication, evolution from previous $4 \mathrm{G}$ networks, has come with an improved set of characteristics that can increase the operational performance of industrial applications. Despite all the advantages, challenges such as interoperability, quality of service, ease of use, reach, cost, and security remain essential goals to be investigated to ensure overall benefit [8]. Please note that the first paragraph of a section or subsection is not indented.

$5 \mathrm{G}$ technology achieves superiority due to its main three cornerstones: massive machine-type communications (mMTC), ultra-reliable and low latency communications (URLLC) Enhanced mobile broadband (eMBB) [3]. In Fig. 2, the communication means can be achieved by $5 \mathrm{G}$ wireless communication in the recycling process environment. The main argument for choosing this is scalability, flexibility, and cost. A wired communication system will need physical installation architecture to increase the cost of a component's change significantly. The communication requirements for 
the recycling process are as follow: (a) update time (process data) $1.5 \mathrm{~ms}$, (b) transmission time (process data) $0.5 \mathrm{~ms}$, (c) distance between logical endpoints $250 \mathrm{~m}$, (d) reliability (redundancy), (e) less than 50 devices connected at the same time. The requirements can be achieved by installing one $5 \mathrm{G}$ base station inside the recycling building to ensure full connectivity between devices and cloud servers and allow remote operability.

\section{Conclusion}

The industrial-scale pyrolysis process to recycle CFRP composite wastes was studied by implementing IoT technology. The study proposes implementing sensors and actuators to collect data from the recycling plant and keeping in mind that the incoming composite wastes to be recycled will have varying composite types and properties. Therefore, the proposed IoT framework will adopt the recycling process conditions according to the composites to unify the standard pyrolysis setup. Furthermore, the methods involved in selecting a suitable IoT platform for the data collection, processing, storage, and visualization of results were also discussed. Overall, implementing the proposed IoT framework will enhance the recycling process adaptability over various CFRP composite waste types. As a result, the proposed IoT framework will facilitate the pyrolysis process into efficient energy utilization, reliability, scalability, and reduce the overall recycling time and cost. Furthermore, the information generated from the data collection and processing using the IoT platform can monitor and maintain the pyrolysis plant. Also, it is capable of influencing current and future recycling business decisions.

\section{References}

1. Karuppannan Gopalraj, S., Kärki, T.: A review on the recycling of waste carbon fibre/glass fibre-reinforced composites: fibre recovery, properties and life-cycle analysis. SN Applied Sciences. 2, 433 (2020). https://doi.org/10.1007/s42452-020-2195-4

2. Gopalraj, S.K., Kärki, T.: A Study to Investigate the Mechanical Properties of Recycled Carbon Fibre/Glass FibreReinforced Epoxy Composites Using a Novel Thermal Recycling Process. Processes 2020, Vol. 8, Page 954. 8, 954 (2020). https://doi.org/10.3390/PR8080954

3. Narayanan, A., Sena, A.S. De, Gutierrez-Rojas, D., Melgarejo, D.C., Hussain, H.M., Ullah, M., Bayhan, S., Nardelli, P.H.J.: Key Advances in Pervasive Edge Computing for Industrial Internet of Things in 5G and Beyond. IEEE Access. 8, 206734-206754 (2020). https://doi.org/10.1109/ACCESS.2020.3037717

4. Gutierrez-Rojas, D., Ullah, M., Christou, I.T., Almeida, G., Nardelli, P.H.J., Carrillo, D., Sant'Ana, J.M., Alves, H., Dzaferagic, M., Chiumento, A., Kalalas, C.: Three-layer Approach to Detect Anomalies in Industrial Environments based on Machine Learning. (2020)

5. Ullah, M., Nardelli, P.H.J., Wolff, A., Smolander, K.: Twenty-One Key Factors to Choose an IoT Platform: Theoretical Framework and Its Applications. IEEE Internet of Things Journal. 7, 10111-10119 (2020). https://doi.org/10.1109/JIOT.2020.3000056

6. Ullah, M., Smolander, K.: Highlighting the Key Factors of an IoT Platform. In: 2019 42nd International Convention on Information and Communication Technology, Electronics and Microelectronics (MIPRO). pp. 901-906. IEEE (2019)

7. Underberg, L., Willmann, S.: Categorization of industrial communication requirements as key to developing

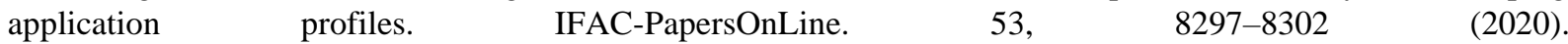
https://doi.org/10.1016/J.IFACOL.2020.12.1919

8. Khaled, Z. El, Mcheick, H.: Challenges and requirements for communication technologies in connected zones. Procedia Computer Science. 155, 27-34 (2019). https://doi.org/10.1016/J.PROCS.2019.08.008 